\title{
BoundarySeer: Visual Analysis of 2D Boundary Changes
}

Wenchao Wu, Yixian Zheng, Huamin Qu, Member, IEEE

Wei Chen, Eduard Gr"oller, Member, IEEE CS and Lionel M. Ni, Fellow, IEEE

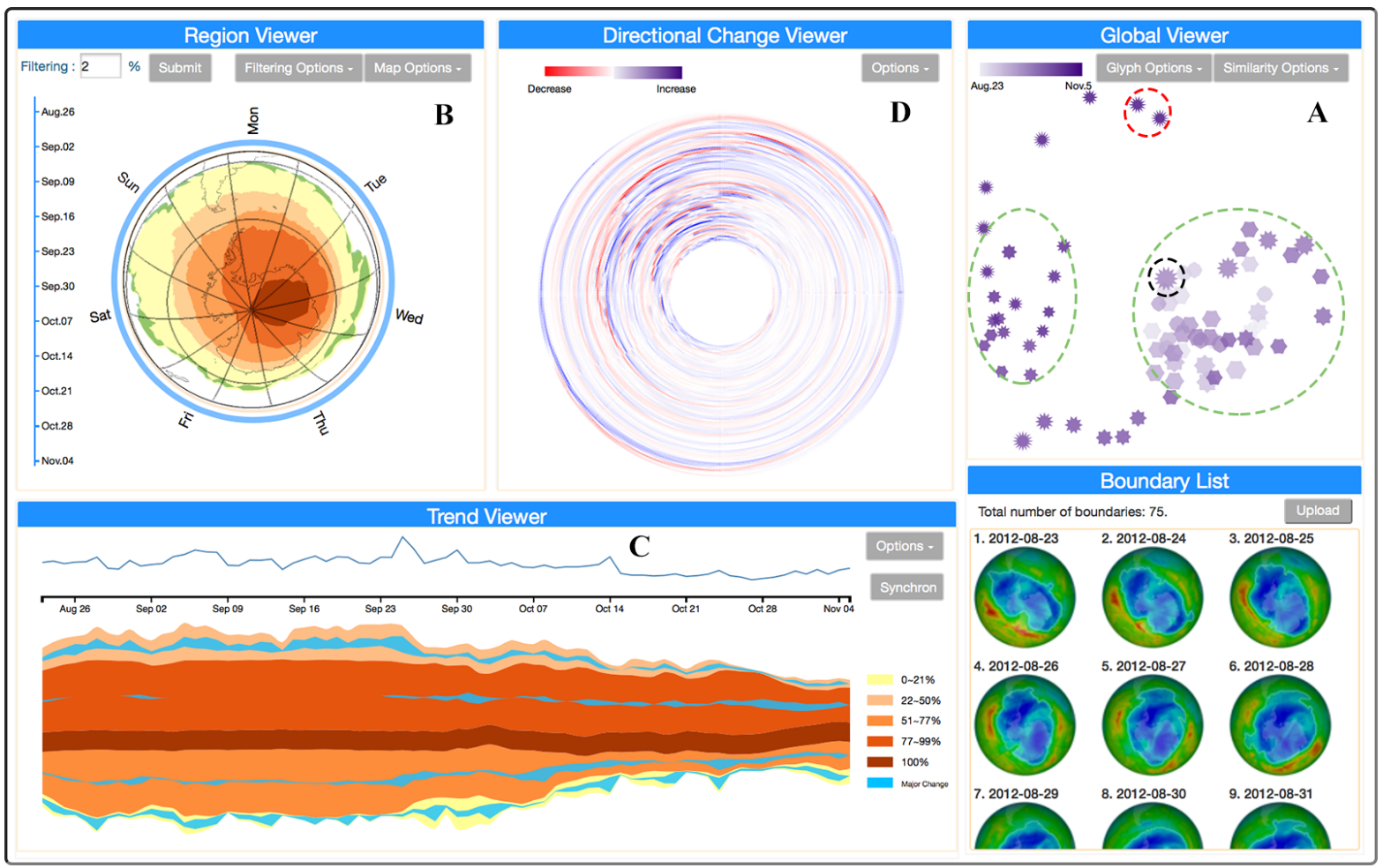

Fig. 1: A visual analytic system used to analyze the boundary changes of the Antarctic ozone hole from Aug. $23^{r d}$ to Nov. $5^{\text {th }}$, 2012. The global viewer shows the similarity of the boundaries and their various attributes such as smoothness, time, and area. The region viewer displays the regions encircled by the boundaries and their stability (e.g., how frequently they are contained in the boundaries). The directional change viewer presents the changes of the boundaries in different directions. The trend viewer reveals the evolution of the area, perimeter as well as the spatial content contained in the boundaries over time.

\begin{abstract}
Boundary changes exist ubiquitously in our daily life. From the Antarctic ozone hole to the land desertification, and from the territory of a country to the area within one-hour reach from a downtown location, boundaries change over time. With a large number of time-varying boundaries recorded, people often need to analyze the changes, detect their similarities or differences, and find out spatial and temporal patterns of the evolution for various applications. In this paper, we present a comprehensive visual analytics system, BoundarySeer, to help users gain insight into the changes of boundaries. Our system consists of four major viewers: 1) a global viewer to show boundary groups based on their similarity and the distribution of boundary attributes such as smoothness and perimeter; 2) a region viewer to display the regions encircled by the boundaries and how they are affected by boundary changes; 3) a trend viewer to reveal the temporal patterns in the boundary evolution and potential spatio-temporal correlations; 4) a directional change viewer to encode movements of boundary segments in different directions. Quantitative analyses of boundaries (e.g., similarity measurement and adaptive clustering) and intuitive visualizations (e.g., density map and ThemeRiver) are integrated into these viewers, which enable users to explore boundary changes from different aspects and at different scales. Case studies with two realworld datasets have been carried out to demonstrate the effectiveness of our system.
\end{abstract}

Index Terms-Boundary change, visual analytics, scatter plot, ThemeRiver, contour map, radial visualization

\section{INTRODUCTION}

- Wenchao Wu, Yixian Zheng, Huamin Qu and Lionel M. Ni are with Hong Kong University of Science and Technology. E-mail: \{wenchao.wu, yixian.zheng\}@ust.hk, \{huamin,ni\}@cse.ust.hk.

- Wei Chen is with State Key Lab of CAD\&CG, Zhejiang University. E-mail: chenwei@cad.zju.edu.cn.

- Eduard Gr"oller is with the Institute of Computer Graphics and Algorithms, Vienna University of Technology and VRVis Research Center, Austria.E-mail: groeller@cg.tuwien.ac.at.

IEEE Symposium on Visual Analytics Science and Technology 2014 November $9-14$, Paris, France

978-1-4799-6227-3/14/\$31.00 @2014 IEEE
We often encounter boundary changes in our daily life. They exist ubiquitously from the reachability of downtown to the diffusion of contamination and from the ozone hole over Antarctica to the territory of a country. Analyzing boundary changes can help to depict the dynamics of an area or the distribution that people are interested in, and to facilitate various analytical tasks, contributing to decision-making and problem solving.

If only a small number of boundaries were concerned, their changes could be analyzed by conventional methods, such as putting them side 
by side to make comparisons, overlaying one onto another to see the difference, or using animations to observe the trends. However, people often need to analyze a large number of boundaries with complex changes in real-world applications. Hence it will be a tedious and time consuming task if conventional analysis methods are used due to the limitation of human short-term memory and severe visual clutter.

To make matters worse, real-world applications often involve a range of different analytical tasks. To analyze a large number of boundaries, people often need to know the overall distribution of boundaries and detect similar boundaries or abnormal changes. Scientists, for example, may need to compare sets of ozone hole boundaries collected over the past years or to find some clusters of boundaries or outliers based on a proper similarity measurement. To understand the impact of the boundary changes and find out the underlying causes behind the changes, the spatial and temporal patterns in the evolution of boundaries and potential spatio-temporal correlations need to be explored and analyzed. Regions under an ozone hole are exposed to harmful radiation and thus we need to know how frequently they are covered by it. As far as reachability is concerned, during different time spans, the one-hour reachable area of downtown varies correspondingly due to different traffic conditions. Urban planners, therefore, may need to find out the periodical changes of reachability of downtown, which can help them improve urban planning and transportation construction. In addition, citizens may be concerned about when they should set off for downtown during different time periods of a day. In addition, detailed directional changes may also be required in the execution of analytical tasks. All these tasks require a quantitative analysis to provide sufficient statistics and precise measurements, as well as intuitive visualizations to depict spatial and temporal patterns of boundary changes.

To address these challenges, in this paper, we propose a visual analytics system, BoundarySeer, which integrates the quantitative analysis of boundaries (e.g., similarity measure, area, smoothness) and intuitive visualizations (e.g., density map, ThemeRiver) into one comprehensive solution for users to explore a large number of boundaries. Our system is based on some well-established visualizations with good scalability and intuitiveness. To perform specific analytical tasks, in particular, to detect potential spatio-temporal correlations in the boundary changes, we further extend these visualization methods such that both spatial and temporal information can be shown in one display. To the best of our knowledge, this is the first comprehensive visual analytics system of the kind that allows users to intuitively analyze a large number of changing boundaries from different aspects and at different scales.

The major contributions of this work are as follows:

- The design and implementation of a comprehensive visual analytics system for exploring the changes in a large number of time-varying boundaries.

- Several visualization designs enhanced with new features including a contour map with adaptive clustering and an extended ThemeRiver with spatial content to reveal spatial and temporal patterns in the boundary changes.

- Two case studies with real-world data to demonstrate the usability and effectiveness of our system.

\section{Related Work}

In this section, we summarize some relevant research work on visualization of boundary changes, comparative visualization, spatial and temporal data visualization and boundary similarity measures.

Visualization of boundary changes Animation is widely used to show boundary changes. However, as demonstrated by Robertson et al. [27], it is not effective for analytical tasks due to the limitation of human short-term memory. There are only a few studies exploiting advanced visualizations to analyze boundary changes. Drocourt et al. [7] investigated the changes in frontal positions (advance/retreat) of 199 marine terminating glaciers in Greenland. A radial visualization is designed to map glacier terminus positions to angular coordinates, and nested rings represent the evolution of the temporal dimension. However, this method fails to show the overall trend and scale of boundary changes, nor can similarity of boundaries at different time points be explored. Furthermore, the method depends on a few discrete data points located on the boundary, which makes it inapplicable to general boundary changes. Visualizing boundary or contour changes is also essential in various applications, such as visualization of medical volume data, where a range of methods $[33,34]$ have been developed. However, most of the approaches are based on position changes of some relatively fixed reference points that may not exist in general boundary changes. In addition, most previous researches focused on topology analysis of smoothly changing boundaries, quite different from the analytical tasks in other applications. In this paper, we address a different and more general issue which has not received much attention from researchers in the field of visualization.

Comparative visualization Visualization of boundary changes falls into the general topic called comparative visualization. Previous work in comparative visualization was systematically surveyed by Gleicher et al. [13]. In this work, there are three major categories of approaches for comparative visualization: juxtaposition, superposition, and explicit encoding. Juxtaposition presents individual objects separately and puts them side by side for comparison, while superposition presents multiple objects in the same coordinate system and overlays one onto another to reveal the difference. Explicit encoding directly encodes differences between objects visually. Various comparative visualization methods have been developed based on these approaches for different comparison tasks, such as comparing large phylogenic trees [25], genetic sequences [26] and other complex data objects [21, 28]. Schmidt et al. [28] proposed a method for visualizing differences and similarities in large sets of satellite images to show changes of a coastline in Indonesia affected by a tsunami in 2004 This method identifies local changes while preserving contextual information by applying cluster analysis techniques. However, it does not allow users to visually explore the temporal trend nor observe how the contour changes over time. In this paper, we propose a visual analytics approach focusing on boundary changes that integrates in-depth analysis and intuitive visualization into a comprehensive solution.

Spatial and temporal data visualization Time-varying boundary data involves both spatial and temporal components. Spatial and temporal data visualization has been extensively studied and many techniques have been proposed. An excellent survey on time-oriented data visualization has been done by Aigner et al. [2]. Line chart and its variants like horizon graph [15] and circular or spiral time axis [38] are the most common ways to show the changes of one attribute over time. Stacked graph [4] is often used to show the changes of multiple attributes over time simultaneously. Geographical information can be displayed with various maps (e.g., heatmap, hotmap $[9,20])$. To detect possible spatio-temporal correlations, we can either encode both temporal and spatial changes into one integrated display $[18,35]$ or show them in multiple linked views [3]. As our system is designed to handle multiple analytical tasks, we adopt a linked view scheme. For multiple linked views, color is often used to help users establish the connection between multiple visualizations. Shanbhag et al. [30] used color components (hue, saturation and brightness) of polygons to analyze and support effective allocation of resources in a spatio-temporal context. Zhao et al. [40] presented Ringmap that used subdivided ring segments with different colors to visualize multiple cyclic activities over time while preserving geographical information. Our system is inspired by these techniques and adopts some similar designs, but encodes different attributes for different applications.

Boundary similarity measures Measuring the similarity of boundaries or shapes has been extensively studied in the field of computer vision and graphics. Measurements in the literature can be categorized into three types: 1) region-based measurements, such as the regional coincidence accuracy [11], the Hamming distance [16]; 2) geometry-based measurements, such as the Hausdorff distance [17], and 3) contour mapping measurements [12, 24, 29]. More details will be discussed in Section 5.1.1. 


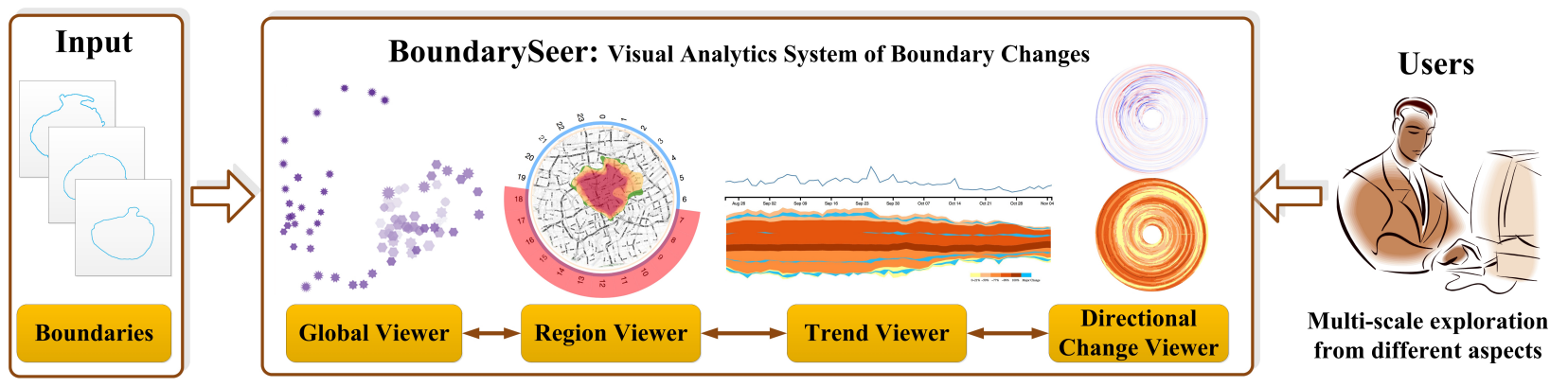

Fig. 2: System framework. After the data is loaded into the system, the global viewer, the trend viewer, the region viewer and the directional change viewer allow users to analyze boundary changes from different aspects and at different scales.

\section{Data And TASk Abstraction}

\subsection{Data Characteristics}

In this paper, we are keenly interested in the kind of boundary change data which is common in many practical applications, such as

- Antarctic ozone hole

- Reachability of downtown area

- Territory of a country

- Land desertification

- Diffusion of contamination

- Calving glaciers in Greenland

Such boundary change data has the following characteristics: First, it involves a large number of boundaries, which makes it a tedious and time-consuming task to view them one by one. Second, unlike "continuous" contour changes in many scientific visualization applications, the boundary changes we investigate are often discrete in the way that a sudden expansion or contraction may take place anytime in any direction. As a result, the conventional analytic approaches widely used in previous work, such as contour tree [5] or superposition [13], are inapplicable. Finally, there are some shared underlying spatial regions within the boundaries (i.e., the boundaries are not floating around). In this paper we focus on time-varying boundaries with each boundary containing only one connected region though the techniques can be generated for cases with multiple connected components.

\subsection{Task Abstraction}

We closely work with three data analysts who investigate topics related to boundary changes in different fields, including urban planning, wireless network and environmental science. They have identified a set of basic and common analytical tasks that are listed as follows:

T.1 The boundary similarity analysis: Analyze boundary similarities and reveal clusters or outliers of boundaries in the data;

T.2 The statistical analysis of boundary attributes: Analyze the overall distribution of common statistics of boundaries (e.g., perimeter, area of regions inside the boundary, smoothness);

T.3 The analysis of regions contained in the boundaries: For a specific geographical region or location, find out how it is affected by the boundary changes (e.g., how often is it contained inside the boundaries during a time period);

T.4 The analysis of temporal patterns: Analyze the trend of the evolution, detect periodical patterns, and find spatio-temporal correlations in the evolution;

T.5 The analysis of directional changes of boundary segments: Investigate details of boundary changes in different directions, such as comparing relative changes in a specific direction over time, or compare relative changes among neighbors.

\section{System OVerview}

Our system is designed based on the information seeking mantra "Overview first, zoom and filter, then details on demand" [31]. It will provide an overview and then allow users to probe the data using zooming and filtering. In addition, as different analytical tasks require different visualizations, our system will provide multiple coordinated or linked visualizations. The BoundarySeer system has four major viewers in all: the global viewer, the region viewer, the trend viewer and the directional change viewer. Fig. 2 shows the framework of our system for visualization of boundary changes.

After the data (a series of boundaries) is input to our system, preprocessing is conducted to compute similarity measures and common boundary attributes such as area and smoothness. A global viewer is firstly provided to give an overview of all boundaries, including the major clusters based on a specific similarity measure and the distribution of some boundary attributes. After that three major viewers are provided for users to explore the data from different aspects and at different scales. The region viewer shows the regions contained in the boundaries and how they are affected by boundary changes (e.g., how frequently they are contained in the boundaries). For further exploration, we enhance the region viewer with temporal information, allowing users to investigate spatial patterns corresponding to a specific time period. The trend viewer shows the temporal trends of boundary changes, in particular, how certain aggregation values change over time. To detect potential spatial-temporal correlations, some spatial information is also incorporated into the trend viewer. Furthermore, if users are interested in detailed changes of boundaries in certain directions, the directional change viewer will visualize the corresponding changes of boundary location and size of the contained area.

The global viewer, the region viewer, the trend viewer and the directional change viewer complement each other and can be linked via user interactions. They work together to provide an effective and comprehensive way to explore boundary changes and allow users to perform a wide range of analytical tasks.

\section{Visualization Design}

In addition to the typical design goals of visualization systems such as effectiveness, efficiency, and aesthetics, we particularly expect our system to be intuitive and have good scalability. BoundarySeer is designed to help analysts from different domains to conduct analytical tasks outlined in Section 3.2. Intuitiveness or simplicity is highly required because our target users are all possible users from different fields working on analytical tasks related to boundary changes, but may not have much background on information technology. Scalability is another key principle determined by the design goals, as the real world data often involves a large number of boundaries.

To satisfy these requirements, we carefully choose the visualization techniques for our system. We only adopt well established visualization techniques such as scatter plots, density maps and ThemeRiver which can easily be understood by users and also have good scalability. To address the specific needs of our problem, these techniques are further extended or tailored for our analytical tasks. In this section, we describe in detail our visualization designs for each viewer. 


\subsection{Global Viewer}

In the global viewer, we want to allow users to grasp the overall characteristics of a series of boundaries and support them to perform the boundary similarity analysis (Task T.1) and the statistical analysis of boundary attributes (Task T.2) outlined in Section 3.2. We first need quantitative methods to make the similarity analysis and compute boundary attributes for all boundaries. Then we need a visualization to present the results to the users. There are standard methods to compute boundary attributes such as area and smoothness. However, the similarity measurements for two boundaries are not trivial. Thus, in this section, we will start with the description on how to compute the similarity between boundaries and then present the visualization design for our global viewer.

\subsubsection{Similarity Measurements}

In order to show an overall distribution of all boundaries, the similarity between each pair of boundaries should be measured based on a proper similarity measurement. Although there are plenty of similarity metrics of boundaries or shapes in the field of computer vision and graphics, it is hard to determine superiority of one metric over another. In reality, it seems that each metric has its own trade-offs. In this part, we briefly explore three major categories of similarity measurements in the literature for the analysis of boundary changes. Several similarity measurements in the three categories are made available for users' choice which can be done depending on different requirements.

Region-based measurements Region-based similarity measurements consider the consistency between the regions contained by two boundaries. For example, the regional coincidence accuracy proposed by Ge et al. [11] is defined as:

$$
P(A ; B)=\frac{\left|R_{A} \cap R_{B}\right|}{\left|R_{A} \cup R_{B}\right|}
$$

where $R_{A}$ and $R_{B}$ are the regions or pixels enclosed by the boundary $\mathrm{A}$ and boundary B. Other examples of region based measurements include the Hamming distance [16], the Negative Rate Metric [14], the Local Consistency Error [22] and the regional Precision-Recall measures [22]. Region-based measurements consider the consistency of regions, but they are not sensitive to spikes, wiggles and major shape differences [24].

Geometry-based measurements Geometry-based measurements evaluate similarity based on geometric properties of boundaries. It is usually some aggregate measure of distance between points on two boundaries. The Hausdorff distance (HD) is a typical geometrybased measurement. However, the Hausdorff distance only looks at the maximum value in distance distributions, and two boundaries having the same largest distance pair will be evaluated as being the same, irrespective of other distances. Most geometry-based measurements share similar characteristics as the Hausdorff distance.

Contour Mapping measurements Contour mapping measurements are in the spirit of elastic matching methods $[10,12]$. They directly align two contours by determining a mapping between point sets of the contours that minimize a matching cost defined on dissimilarity. To avoid the problem caused by the aforementioned two measurements, the order of mapping must be monotonic, however, not strictly monotonic. This allows the mapping to be one-to-one, one-to-many, or many-to-one, which is also called bimorphism [32].

This kind of measurements has been shown to be sensitive to shape irregularity and most consistent with human perception based on comprehensive experiments [24]. The contour mapping measurement, we adopt, is described as follows:

Define a mapping sequence $S=s_{1} s_{2} \ldots s_{k}$ between point sequences of boundary $A=a_{1} a_{2} \ldots a_{n}$ and $B=b_{1} b_{2} \ldots b_{m}$. All points in A must be mapped to at least one point in $\mathrm{B}$ and vice versa. In addition, for two distinct pairs corresponding to two mappings in $\mathrm{S},\left(a_{i_{1}}, b_{j_{1}}\right)$ and $\left(a_{i_{2}}, b_{j_{2}}\right)$, we have $i_{1}<i_{2} \Rightarrow j_{1} \leq j_{2}$. Thus the order is preserved.

The cost of sequences $\gamma(S)$ is defined by the sum of Euclidean distances between the points $\gamma\left(s_{i}\right)$ :

$$
\gamma(S)=\sum_{i=1}^{k} \gamma\left(s_{i}\right)
$$

The mapping distance $\delta(A, B)$ then is defined as the minimum cost of mapping $\mathrm{A}$ and $\mathrm{B}$ :

$$
\delta(A, B)=\min _{S} \gamma(S)
$$

Distance $\delta(A, B)$ can be efficiently computed by dynamic programming [19, 24].

\subsubsection{Visual Encoding Schemes}

Now that we have the similarity value between any two boundaries and also the statistical values such as area for each boundary, we need a visualization to show the results to the users. In our system, we adopt a scatter-plot display with each point representing a boundary and relative distance encoding the similarity. Meanwhile, to show the overall distribution of attribute values, we extend the point-based display with an attribute glyph to encode the attribute values of users' interests. We now describe the point-based display and the glyph in turns.

Scatter Plot Encoding As described in Section 5.1.1, the overall distribution of a series of boundaries should be measured by similarity measurements between all pairs of boundaries. Thus, the similarity of each boundary in context is represented as a high-dimensional vector $\left(d_{i, 1}, d_{i, 2}, \ldots, d_{i, i-1}, d_{i, i+1}, \ldots, d_{i, n}\right)$ where $n$ is the number of all boundaries. $d_{i, j}$ represents the value of similarity measurement between boundary $\mathrm{i}$ and boundary $\mathrm{j}$.

Through Multidimensional Scaling (MDS), the boundary corresponding to the vector $w_{i}=\left(d_{i, 1}, d_{i, 2}, \ldots, d_{i, i-1}, d_{i, i+1}, \ldots, d_{i, n}\right)$ can be mapped to a point in a scatter plot. The distance between each pair of points encodes the similarity between corresponding pairs of boundaries. Through the scatter plot layout, users can easily observe the clusters of similar boundaries and outliers (Task T.1).

Boundary Glyph The scatter plot view can reveal the similarities between boundaries. However, users may also wish to have an overall understanding of the distribution of some common statistics of the boundaries and their evolution over time (Task T.2). For this, we design a boundary glyph (see Fig. 3) and replace each point in the boundary graph with a boundary glyph that encodes various attributes of the boundary.

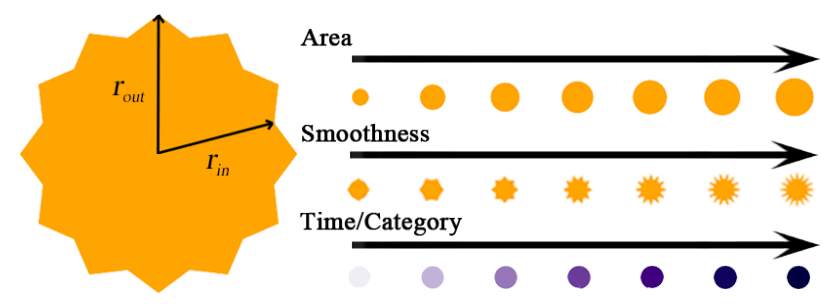

Fig. 3: Boundary glyph to encode the area, smoothness, and the time of a boundary.

Users are often interested in the time, area, and smoothness of a boundary. It is a natural way to encode the area of a boundary using the size of the glyph and the smoothness of the boundary with the shape of the glyph. The encoding scheme is illustrated in Fig. 3. We design a glyph that consists of an inner circle and an outer star. To differentiate boundaries with different areas of the interior regions, we modify the radius of inner circle $r_{\text {in }}$ of the glyph. Intuitively, the larger the radius, the larger the area size. To present boundaries with different smoothness, the geometry of the glyph is employed. We use the number and the sharpness of spikes to encode the level of smoothness. A boundary with a low level of smoothness will have more and sharper spikes in the outer star. The sharpness of spikes is defined as follows:

$$
\frac{r_{\text {out }}-r_{\text {in }}}{r_{\text {in }}} \sim f_{\text {smoothness }}
$$


Equivalently, the outer radius $r_{\text {out }}$ of the glyph can be determined as follows:

$$
r_{\text {out }}=r_{\text {in }}+f_{\text {smoothness }} \cdot r_{\text {in }} \quad \text { s.t. } f_{\text {smoothness }} \in(0,1.0]
$$

Moreover, to encode temporal information in the global viewer, we can also use the color of the glyph to encode different times of the day [39].

For some applications, the anisotropy, concavity, and complexity information of boundaries is also important for analytical tasks. The boundary glyph can be further extended to encode these attributes. For example, the outer star might become asymmetric to encode anisotropy information. The inner circle might be transformed into a simple concave shape to encode concave boundaries.

To reduce visual clutter, not all of these encoding schemes need to be shown simultaneously. Users can configure the system to decide which attributes they want to see in the global viewer.

\subsection{Region Viewer}

In this section, we describe the design of the region viewer. The goal of the design is to visualize the spatial patterns of boundary changes for further exploration and analysis. In this viewer, we focus on the impact of the boundary changes on different locations.

\subsubsection{Design Rationale}

As described in Section 3.1, we focus on boundaries which have some common underlying regions. Some important analytical tasks (Task T.2, T.3) involve the study of characteristics of the regions and how they are affected by boundary changes. Thus, it is desirable to provide a viewer that can show the geographical information of these regions and reveal the "stability" of the regions with respect to the boundary changes.

A map is preferably used to show geographical information and some other information overlaid on top of it. To analyze the impact of boundary changes on different locations, the aggregated region inside the boundary is used to compute the stability of each location which has ever been contained by a boundary. Then a heat map is generated to present the spatial change scale of boundaries and the stability of different locations. This provides an intuitive and explicit spatial information to the users. Moreover, locations are grouped into different clusters with an adaptive clustering algorithm to form a contour map for further exploration. This also facilitates discovery of spatiotemporal correlations in the trend viewer (see Section 5.3). Users can further brush by time or by stability level to filter the data and generate heat maps and contour maps for subsets of the data that they are interested in.

\subsubsection{Visual Encoding Schemes}

To provide enough information to explore spatial patterns of boundary changes without causing visual clutter [8], we design an interactive viewer consisting of two kinds of maps with an optional layer and optional time axes.

Heat Map with Kernel Density Estimation First, the stability of different locations or regions should be identified. By stability, if given a specific geographical region or location, users can easily find out how many boundaries contain it, which is a common spatial pattern for analytical tasks (Task T.3) of boundary changes. We rasterize the region that may be contained by boundaries into grids. Then stability $S_{i, j}$ of grid $(i, j)$ is computed using the following formula:

$$
S_{i, j}=-c \cdot \ln \frac{\sum_{l=1}^{N} x_{i, j}^{l}}{N}
$$

where

$$
x_{i, j}^{l}= \begin{cases}1 & \text { if grid }(i, j) \text { is in the } l t h \text { boundary } \\ 0 & \text { otherwise }\end{cases}
$$

and $\mathrm{N}$ is the total number of boundaries, and $\mathrm{c}$ is a constant to normalize the stability values.
Kernel density estimation (KDE) can then be used to derive a smooth representation of the density distribution on the plane. In this way, we can form a heat map to reveal the overall spatial stability of boundary changes. Fig. 4a shows the heat map with red areas representing regions of high stability while white areas representing regions of relatively low stability.

Contour Map with Adaptive Clustering Based on the initial heat map, we further cluster different locations with similar stability into several groups. This not only enhances the analysis of spatial patterns but also facilitates the temporal exploration in the trend viewer. To further support exploration and analysis, we apply an adaptive Kmeans clustering algorithm which automatically determines the most suitable and reasonable number of clusters for visualization. Considering legibility and aesthetics, the upper bound of the number of clusters should be ten in order to enable reliable identification [37], while the lower bound is set to three so that enough details will be provided for analysis. The adaptive clustering is performed based on index- $\mathfrak{I}$, a more consistent and reliable cluster validity index compared to some other common indices (e.g., Davies-Bouldin Index) to indicate the appropriate number of clusters [23], which is defined as follows:

$$
\mathfrak{I}(K)=\left(\frac{1}{K} \times \frac{E_{1}}{E_{K}} \times D_{K}\right)^{p}
$$

where $\mathrm{K}$ is the number of clusters, and the power $\mathrm{p}$ is used to control the contrast between the different cluster configurations. Here,

$$
E_{K}=\sum_{k=1}^{K} \sum_{j=1}^{n} u_{k j}\left\|x_{j}-z_{k}\right\|
$$

and

$$
D_{K}=\max _{i, j=1}^{K}\left\|z_{i}-z_{j}\right\|
$$

$\mathrm{n}$ is the total number of points in the dataset, $\left[u_{k j}\right]_{K \times n}$ is a partition matrix for the data, and $z_{k}$ is the center of the $k^{\text {th }}$ cluster. The value of $\mathrm{K}$ for which $\mathfrak{I}(K)$ is maximized is considered to be the most suitable number of clusters.

After clustering, the different clusters are then filled with sequential colors using the same encoding scheme as in the heat map. The resulting contour map (Fig. 4b) is a visual abstraction of the spatial characteristics of boundary changes for analysis.

Brushing by Time and/or Stability The heat map and contour map can show the overall distribution of boundaries according to their "stability" for all the available data. Our system further allows users to select a specific time period. Automatically updated maps are generated reflecting the boundaries in response to the corresponding timeframe. A circular time axis for periodical time selection (e.g., 8 a.m. - 9 a.m., Monday - Wednesday) [40] and a horizontal (or vertical) time axis for linear time selection (e.g., Nov. $8^{\text {th }}$ ) are provided. Users can directly brush the axes and the maps will be updated accordingly. Sometimes users may want to explore specific regions or locations with a certain stability (e.g., the locations with stability lower than $5 \%$ of the average stability). They only need to input a stability number (e.g., five) and then the corresponding regions will be highlighted in the map. Figures $4 \mathrm{c}$ and $4 \mathrm{~d}$ shows the contour maps after brushing the daytime of two different days.

\subsection{Trend Viewer}

In this section, we describe the design of the temporal viewer that reveals the temporal patterns of boundary changes and potential spatiotemporal correlations in the boundary evolution.

\subsubsection{Design Rationale}

As boundary change data is a typical type of time-varying data, temporal patterns are another important aspect for analysis. Therefore, the trend viewer is mainly designed to show temporal patterns in boundary changes. However, for many practical applications, the users need 


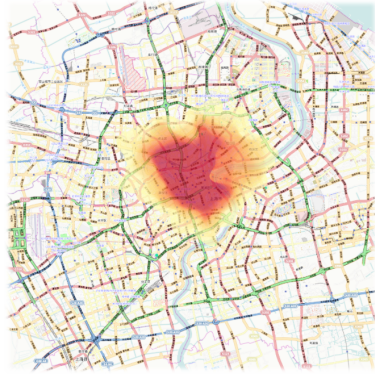

(a)

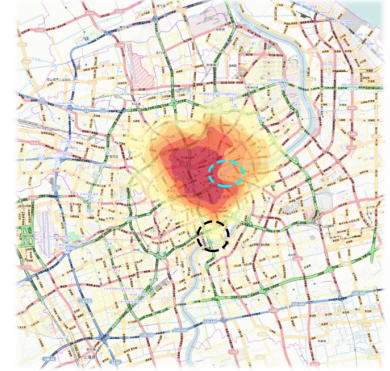

(b)

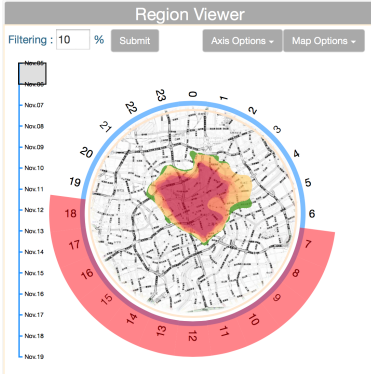

(c)

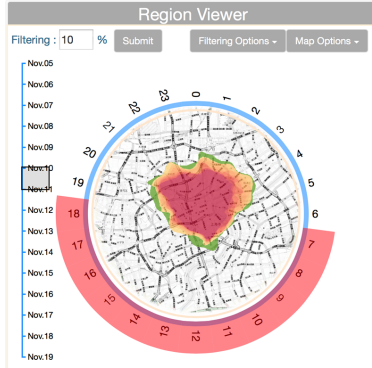

(d)

Fig. 4: Region viewer: (a) The heat map shows the overall spatial characteristics of boundary changes. The darker the color, the more often the corresponding region has been contained by the boundaries; (b) The contour map shows region clusters with different levels of stability facilitating the discovery of spatio-temporal correlations (see Section 6.2); (c) Users can brush by time and stability level to show the heat map or contour map with the stability falling into the specified level during the selected time period (7 a.m. -6 p.m. on Nov. $5^{\text {th }}$, 2007 with stability less than $10 \%$ in this figure); (d) The contour map for 7 a.m. -6 p.m. on Nov. $10^{\text {th }}$, 2007 with stability less than $10 \%$.

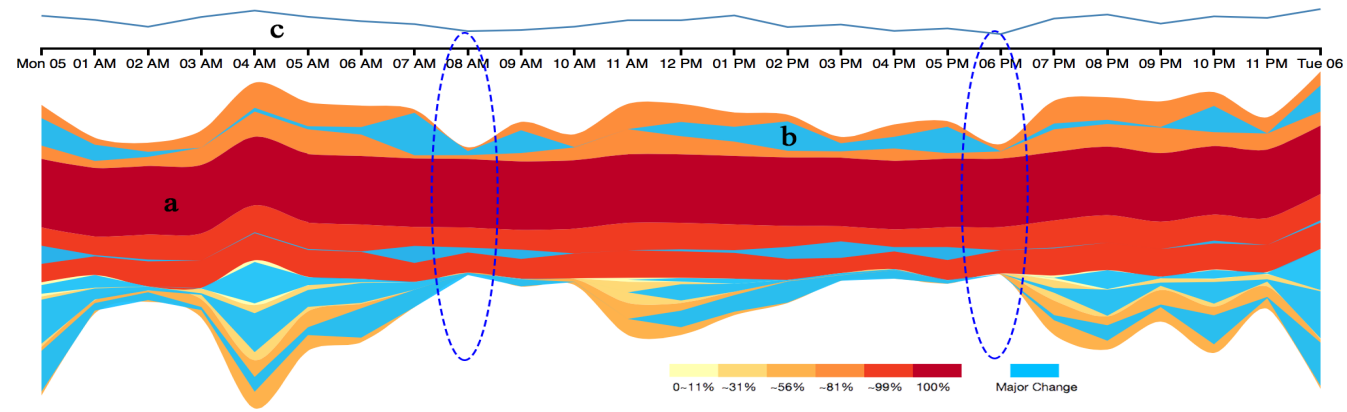

Fig. 5: Trend viewer: (a) ThemeRiver: each layer of the ThemeRiver represents a category of spatial regions based on clusters with different stabilities generated in the region viewer. The same color map has been used to encode the categories both in the ThemeRiver of the trend viewer and the heat map of the region viewer; (b) Threads show major changes of a region in adjacent timeframes. The width of the threads is proportional to the amount of major changes for each cluster; (c) Trend curve shows the perimeter changes of boundary.

to further analyze temporal and spatial correlations in order to investigate various temporal patterns of boundary changes (Task T.3, T.4, T.5). Thus, in this viewer, we want to combine the spatial and temporal information of boundary changes to facilitate discovery of temporal patterns based on potential spatio-temporal correlations.

In order to integrate spatial information into temporal exploration and detect possible correlations (Task T.3), we utilize the clusters of locations generated for the contour map of the region viewer. Furthermore, we introduce an extended ThemeRiver with embedded spatial information and an integrated trend curve to show the evolution of area of boundaries as well as other attributes.

\subsubsection{Visual Encoding Schemes}

The ThemeRiver provides an intuitive methodology to integrate temporal characteristics of area changes of boundaries. A composite style is employed: the changes of area size in different region clusters are depicted using ThemeRiver, while more details of changes are encoded as threads placed on the layers in the ThemeRiver, given that details complement and facilitate temporal and spatial pattern analysis of boundary changes. The composite visual design that integrates various types of spatio-temporal data will make identifying temporal and spatial correlation patterns more efficient as compared to depicting data separately [36]. Fig. 5 shows a ThemeRiver based on our design.

Layers in ThemeRiver Each layer corresponds to a region cluster with similar stability. The height of each layer is proportional to the total area of regions belonging to the cluster. Given that changes in different region clusters are directly comparable, the accumulated height can reveal the overall area change of the boundaries over time.
Color intensity encodes the stability of the region clusters. Clusters with higher stability will be assigned higher values.

Threads On each layer of ThemeRiver, a highlighted thread is drawn to depict major changes of the corresponding regions in adjacent timeframes, thus changes with similar area size but different locations can be differentiated. The width of the threads is proportional to the amount of major changes for each cluster. The thicker a line is, the more significant is the corresponding change.

Trend Curve Additionally, the ThemeRiver can be augmented with a trend curve which can show the changes of another aggregation value such as perimeter over time. It shares the temporal axis with the ThemeRiver so that users can intuitively analyze correlations between different attributes of boundaries.

\subsection{Directional Change Viewer}

In this section, we describe the directional change viewer that visualizes detailed changes of a boundary in different directions. It facilitates users to explore more details of boundary changes in certain directions.

Radial visualizations, such as ring maps and polar maps, are commonly used to encode values in certain directions [38]. Inspired by ring maps, we also adopt a radial layout to represent a series of boundary changes simultaneously. We create multiple sub-rings and assign the initial boundary change to the inner most ring and the final one to the outer most ring. Each sub-ring is separated into a number of sectors, each representing a specific direction. Changes in a geographic direction can thus be intuitively revealed by the corresponding sector.

Fig. 6 shows the directional change viewer of our system. We provide two different encoding schemes to effectively convey the boundary directional area changes and position changes respectively. We call 
the circle representing the area of directional changes of a boundary the area circle, and the circle representing the position of directional changes the position circle. The encoding schemes are described as follows:

Area Circle To visualize detailed area changes in different directions, we use the contrasting colors, red and blue to fill the sectors in each sub-ring of the radial layout to encode expansion or contraction of a boundary in a certain direction from one time step to the next (see Fig. 6a) . Color intensity encodes the amount of area expansion or contraction of a boundary.

Position Circle To visualize changes of the boundary position in different directions, each sector in a sub-ring of the radial layout is colored based on the frontal location of the corresponding boundary in that direction (Fig. 6b). The color can be chosen based on the clustering result generated in the contour map of the region viewer (refer to Section 5.2).

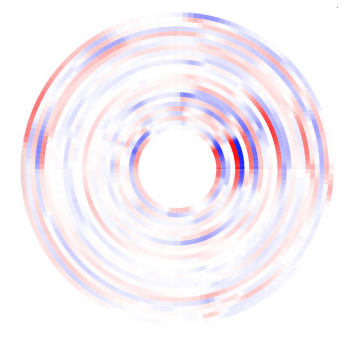

(a)

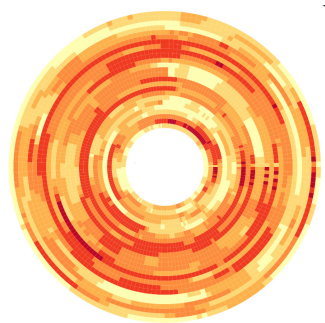

(b)
Fig. 6: Directional change viewer: (a) Area Circle shows area changes in different directions; (b) Position Circle shows position changes of boundary segments in different directions.

\subsection{Interactions}

Our system also provides users with rich interactions to help them perform a comprehensive analysis of boundary changes. Besides typical user interactions like filtering and brushing, BoundarySeer also supports the following interactions:

Configuration Users can configure our system to choose default views (e.g., heat map or contour map for the region viewer; area circle or position circle for the directional change viewer) and parameters for different viewers. For example, in the global viewer, users can choose which attributes (e.g., area, smoothness, time) shall be shown on the display.

Connecting Functionality is provided for further exploration of temporal information in the global viewer. If users double click a point of interest, the other temporally related points will be highlighted with distinct colors based on the temporal information.

Linking The system supports automatic linking among the four proposed viewers. For example, if a segment of the ThemeRiver in the trend viewer is brushed, the corresponding points in the global viewer and related coverage area in the region viewer will be highlighted, and the directional change viewer will be updated accordingly.

\section{Case Studies}

In the following, we demonstrate the usage and effectiveness of our visual analysis system through two use cases: the boundary changes of the ozone hole over Antarctica and the reachability of downtown in Shanghai. The preprocessing took about 2 minutes for the ozone hole data and 8.5 mintues for the Shanghai trajectory data. After that, the system can run in real time.

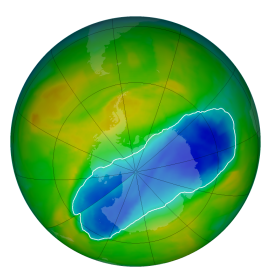

(a)

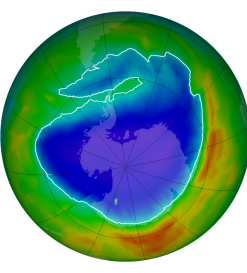

(b)

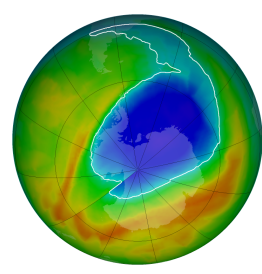

(c)
Fig. 7: Ozone hole boundaries. (a) The outlier boundary on Nov. $3^{\text {rd }}$, 2012; (b) The boundary of the ozone hole on Sep. $25^{t h}$, 2012; (c) The boundary of the ozone hole on Oct. $14^{\text {th }}, 2012$.

\subsection{Case Study 1: Ozone Hole}

The first case study illustrates how BoundarySeer can help environmental scientists to interactively analyze boundary changes of the ozone hole over Antarctica and detect spatial and temporal patterns in the changes. We have collected 75 boundaries of the Antarctic ozone hole for about two months (Aug. $23^{\text {rd }}$ to Nov. $5^{\text {th }}, 2012$ ) from NASA's database [1]. We applied our system to the data and the results are shown in Fig. 1.

We first examined the overall characteristics of the ozone hole boundaries in the global viewer, where we could clearly see that there are two major clusters (two groups of points highlighted in green circles in Fig. 1A) of boundaries based on the contour mapping similarity measurement (Task T.1). We could also identify some outlier boundary points (highlighted in a red circle in Fig. 1A) which had quite different shapes from other boundaries, like a paramecium (Fig. 7a). Afterwards, we tried to find out the distributions of various statistical values of boundaries by inspecting boundary glyphs. By observing the size of points, we found the two clusters to be quite different in areas. By observing the shape of the glyphs which encodes the smoothness, we identified a very rough boundary in the middle of the scatter plot (highlighted in a blue circle in Fig. 1A) corresponding to the boundary of Sep. $25^{\text {th }}, 2012$ which is unique with its tentacle-like stretched area (Fig. 7b) (Task T.2).

Afterwards we used the region viewer to examine the regions contained in these boundaries (Task T.3). By studying the contour map with adaptive clustering in the region viewer (Fig. 1B), we observed five region clusters with different levels of stability. We can easily determine that the South Pole is not the center of a constantly covered region. Also during the investigated period, the ozone hole covers almost the whole of Antarctica with quite high stability, which may lead to intense ultraviolet radiation, causing harm to the creatures living there.

Next, we moved on to explore temporal patterns using the trend viewer (Fig. 1C) (Task T.4). Generally speaking, the area of the ozone hole was stable from September to October in 2012, however, with a gradual contraction in October. Through the trend curve we saw the perimeter of the boundary fluctuating over time in a quite different pattern from the one in the ThemeRiver. The peaks of the trend curve in the ThemeRiver denote boundaries most likely to have unique shapes, such as the one on Oct. $14^{\text {th }}, 2012$ (Fig. 7c). Then we analyzed the data at the layer level of the ThemeRiver. In the top-most layer, the stability level of that location cluster is $22 \%$ to $50 \%$ (Fig. 1C). Before October, the area basically remained the same, but based on the augmented highlighted thread in this layer, we can conclude that the actual corresponding region of that cluster changed dramatically.

Finally, we used the directional change viewer to get more details of ozone hole boundary changes in specific directions (Task T.5). By observing the area circle encoding area changes (Fig. 1D) in the viewer, we found that significant changes of the boundary took place between 30 to 80 degrees west longitude during that period, corresponding to these directions in the top left corners of the radial layout, which maybe the most unstable directions of the ozone hole. 


\subsection{Case Study 2: Reachability of Downtown}

In the second case study, we analyzed the changes in the boundary of an area within half-hour reach from the People's Square, a metropolitan transportation hub as well as a famous scenic spot in downtown Shanghai. We obtained 336 boundaries based on over 20 million GPS records collected from around 6,000 taxis in Shanghai for two weeks, from Nov. $5^{\text {th }}$ to Nov. $18^{\text {th }}, 2007$.

By observing the global viewer (Fig. 8a) for overall characteristics of the data, we found that there seemed to be no clear clusters. However by examining the colors of the points, we could see that points in blue corresponding to 7 a.m. and points in red corresponding to 8 a.m. are in two separate clusters (Fig. 8a). This indicates a significant change of traffic conditions during that period. As shown in Figures $8 \mathrm{c}$ and $8 \mathrm{~d}$, the boundary of reachability on Nov. $15^{\text {th }}$ contracted a lot from 7 a.m. to 8 a.m. in multiple directions, especially in the east. We could also identify some outlier boundary points (highlighted by a green arrow in Fig. 8a) at the bottom of the scatter plot, indicating a unique reachable area (Fig. 8b) at 9 a.m. on Nov. $15^{\text {th }}, 2007$.

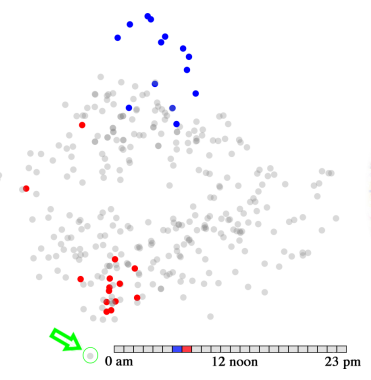

(a)

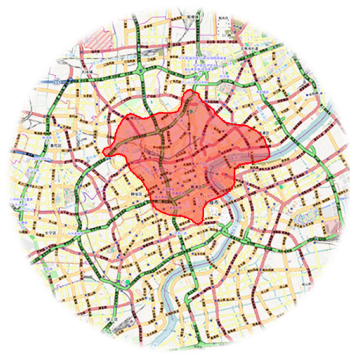

(c)

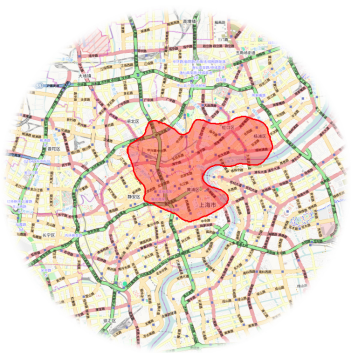

(b)

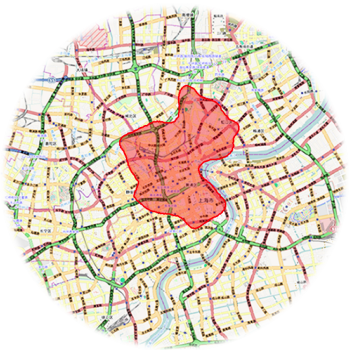

(d)
Fig. 8: (a) The global viewer shows that points in red and points in blue belong to two different clusters. The arrow points to an outlier boundary; (b) The outlier boundary at 9 a.m. on Nov. $15^{\text {th }}, 2007$ indicates a unique reachable area; (c) The boundary at 7 a.m. on Nov. $15^{\text {th }}, 2007$ which is quite different from the boundary at 8 a.m.; (d) The boundary at 8 a.m. on Nov. $15^{\text {th }}, 2007$.

The region viewer in Fig. 4a shows the areas within half-hour reach from the People's Square during that period (Task T.3). They are then grouped into six clusters according to different levels of stability (see Fig. 4b). In Fig. 4b, it is found that areas with high stability were mostly located in Puxi, whereas on the other side of the Huangpu River, the stability decreased significantly, especially for the area highlighted in the cyan circle in Fig. 4b. The significant decrease of stability in that area was due to constant jams of the traffic approaching the Yan An Road Vehicular Tunnel that passes under the Huangpu River, connecting Puxi and Podong. Moreover, we observe the reachable area extended sharply in the area highlighted in the black circle in Fig. 4b. The extended reachability was made possible thanks to the $\mathrm{Lu} \mathrm{Pu}$ Bridge, a newly built bridge standing astride the Huangpu River in 2004.

The trend viewer in Fig. 9a illustrates the temporal patterns (Task
T.4) of reachability in downtown Shanghai for the first week of that period. Generally, the reachable areas contained by the boundaries for weekends are larger than those for weekdays. The peaks mainly appear before dawn, when there are few cars in downtown. The patterns in weekdays (the first five cells) and those at weekends (the last two cells) are different.

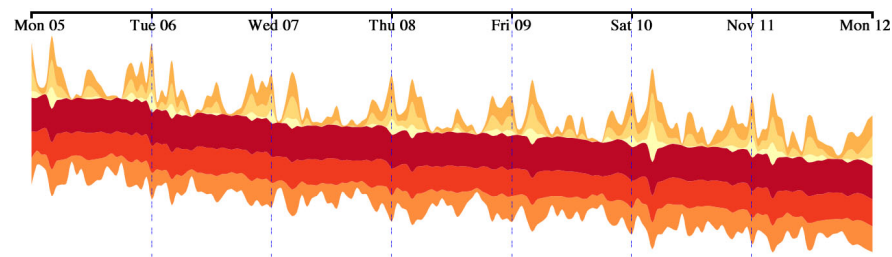

(a)

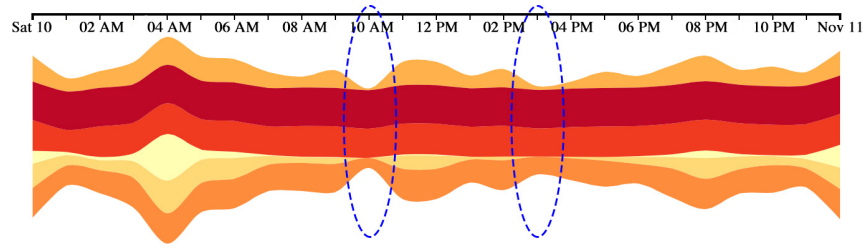

(b)

Fig. 9: The trend viewer for the reachability of downtown in Shanghai: (a) The ThemeRiver for the period from Nov. $5^{\text {th }}$ to Nov. $11^{\text {th }}$, 2007 shows different temporal patterns for different days (separated by dotted lines); (b) The ThemeRiver for Nov. $10^{t h}, 2007$ (Saturday) with two valleys at 10 a.m. and 3 p.m. marked with blue indicates two traffic peaks.

Fig. 10a and Fig. 10b show the directional change viewer consisting of two circular plots to help users investigate changes in different directions (Task T.5). In the area circle, we observe frequent and significant changes to the east of the People's Square (highlighted in a green box in Fig. 10a), corresponding to the aforementioned Yan An Road Vehicular Tunnel. In addition, we also see several segments to appear periodically with intense color in the directions highlighted by a black box in Fig. 10a. After checking the temporal information of these segments, we found that they appeared right after midnight, which may indicate heavy traffic flows after closure of shops in the vicinity of the People's Square. In the position circle (Fig. 10b), we observe obvious dark sub-rings separated from each other by bright sub-rings with almost equal width, which demonstrates explicit periodical movements of the boundary segments in different directions. Moreover, we can see that there are darker segments highlighted by a blue circle in Fig. 10b, which indicates worse traffic conditions to the east of the People's Square.

Furthermore, users can perform exploration through interactions among the linked viewers. For example, if users want to compare different temporal and spatial patterns for weekdays and weekends, they can simply brush on the ThemeRiver in the trend viewer. A new ThemeRiver and the corresponding region viewer and directional change viewer will be generated for the selected period. Fig. 5 and Fig. 9b show two ThemeRivers for Nov. $5^{\text {th }}$ (Monday) and Nov. $10^{\text {th }}$ (Saturday). By comparing two ThemeRivers, we can find two different temporal patterns in weekdays and at weekends. Two valleys (highlighted in blue circles in Fig. 5) correspond to two traffic peaks in weekdays. They appear in different time periods from those at weekends (highlighted in blue circles in Fig. 9b). The traffic peaks occur at 8 a.m. and 6 p.m. on Monday, while they happen at 10 a.m. and 3 p.m. on Saturday. In the region viewer, users can further brush on the circular axis to investigate spatial patterns for different periods. Fig. $4 \mathrm{c}$ and Fig. $4 \mathrm{~d}$ show two contour maps for 7 a.m. -6 p.m. on Nov. $5^{\text {th }}$ and Nov. $10^{\text {th }}$ respectively. The figures indicate that the traffic condi- 
tion was quite different during daytime of weekdays and weekends. In the directional change viewer, the position circle (Fig. 6b) for Nov. $5^{\text {th }}$ (Monday) has more dark segments than the one for Nov. $10^{\text {th }}$ (Saturday) (Fig. 10c). This shows that the area within half-hour reach from the People's Square on Monday is generally smaller than on Saturday. Furthermore, the area circles for Nov. $5^{\text {th }}$ (Fig. 6a) and Nov. $10^{\text {th }}$ (Fig. 10d) show that although the patterns for weekdays and weekends are different, changes always occur in certain directions. The transportation system in these directions might need to be improved.

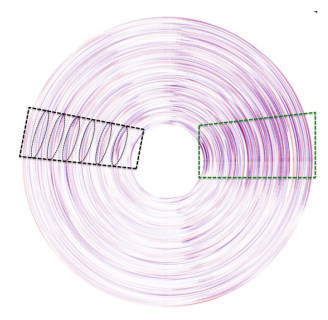

(a)

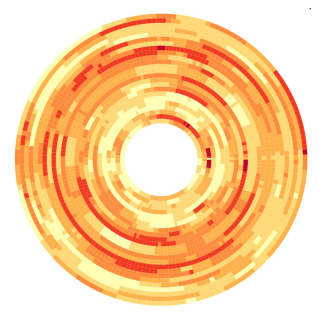

(c)

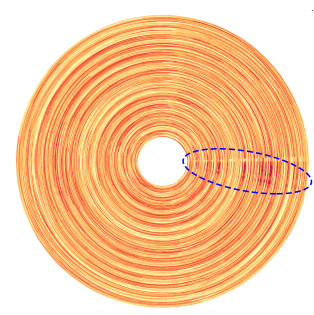

(b)

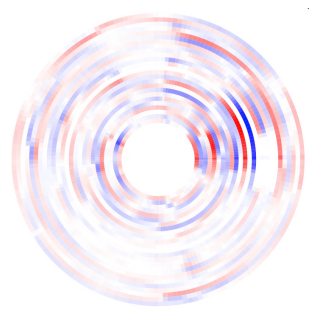

(d)
Fig. 10: The directional change viewer for the reachability of downtown in Shanghai from Nov. $5^{\text {th }}$ to Nov. $10^{\text {th }}, 2007$ : (a) The area circle for the period indicates typical directions where significant (highlighted in green box) and periodic (highlighted in black box) boundary changes took place; (b) Darker segments (highlighted by a blue circle) in the position circle indicates worse traffic conditions during the period; (c) The position circle for Nov. $10^{\text {th }}$, a Saturday, has more bright segments, indicating better reachability on this day; (d) The area circle for Nov. $10^{\text {th }}$ indicates typical directions where significant boundary changes took place during that day.

\subsection{Expert Interview}

To evaluate the effectiveness of BoundarySeer, we demonstrated our system to a domain expert who has worked on the change of the polar ozone sphere for more than ten years and conducted a one-on-one interview with him to collect his feedback. The domain expert was impressed with the visual design. He commented that the system could intuitively reveal the complex change of the ozone hole. To his knowledge, the system is the first comprehensive system that allows users to intuitively analyze a large number of changing boundaries of the ozone hole from different aspects and at different scales. The domain expert also appreciated the linked viewers in our system. They help to demonstrate the stability of the Antarctic ozone hole (based on the temporal viewer) and frequent changing directions (based on the directional change viewer) caused by the stable geographical structure in Antarctica. The findings obtained by our system are backed up by the facts he has already known, but the way we obtained these findings impressed him. Finally, the expert recommended to encode other information, especially weather, in the system to help analyze the causes of ozone hole changes, which is one of the important problems in his field.

\section{Discussion}

The case studies demonstrate the advantages of our system to explore boundary changes at different scales and from different aspects. With BoundarySeer, potential spatio-temporal correlations, which facilitate the discovery of temporal and spatial patterns, can be detected intuitively. The visualization schemes adopted in our system, such as pixel-based techniques, density map, and ThemeRiver, can be readily comprehended by users with different backgrounds.

In addition, our approaches can be easily extended to handle topological changes. The ThemeRiver in the trend viewer, for example, can be further extended to encode branch split and merge to represent topology changes, similar to the scheme used in TextFlow [6]. Then other viewers can be generated for each connected region separately.

Our research is still in progress. Weaknesses have been observed and will be addressed in the future. Firstly, we assume all boundaries to share some common underlying regions that have good stability on the whole. Thus, our method cannot handle floating boundaries at this stage. Registration is required for floating boundaries prior to the application of our system. Secondly, as we use color to encode different region categories, only 8 to 12 categories can be shown in one display. Thirdly, although our system is designed for boundaries containing one or multiple connected components, it will encounter problems if there are hundreds of small connected components, which is beyond the scope of this work. Finally, our system will be faced with scalability issues if tens of thousands of boundaries need to be analyzed. More levels of detail and abstraction can be introduced to handle this problem. For example, if thousands of rings are shown in the directional change viewer, we can cluster similar rings into super rings and encode the statistical information of these super rings. When users click on a super ring of interests, the contained rings will be expanded and visualized for further analysis. We will pursue more analysis and exploration of general boundary changes based on computational geometry and data mining techniques to further improve our work.

\section{Conclusion}

In this paper, we have studied the subject matter of boundary change and presented BoundarySeer. It is a visual analytic system consisting of four major viewers, to facilitate the general analytical tasks dealing with boundary changes. Some well established visualization techniques such as scatter plot, heat map and ThemeRiver are extended and integrated into our system. Aside from improving the scalability, they also increase the legibility of the system. We have proposed several novel visual encoding schemes such as the region viewer and trend viewer to detect potential spatio-temporal correlations for further exploration of temporal and spatial patterns, the global viewer to show overall distribution and the directional change viewers to reveal the evolution of a boundary in different directions. Furthermore, we have investigated the usability and effectiveness of our system by case studies based on two real world datasets. The advanced visual analytic techniques combined with intuitive user interactions allow users to interactively explore and analyze boundary changes from different aspects and at different scales. Our method has a wide range of applications and can be used to analyze the changes of skin cancer boundaries, iso-thermal lines in 2D weather maps, and so on.

There are multiple avenues for future work. We plan to extend our BoundarySeer system to cope with topological changes and apply it to boundary change datasets in other application domains. We intend to collect more feedback from end users and conduct controlled experiments to further improve our system.

\section{ACKNOWLEDGMENTS}

The authors thank the anonymous reviewers for their valuable comments. This research was supported in part by the National Basic Research Program of China (973 Program) under Grant No. 2014CB340304, the Major Program of National Natural Science Foundation of China (61232012) and the Austrian Science Fund (FWF) in the scope of project P 24597-N23 (VISAR). 


\section{REFERENCES}

[1] NASA Ozone Watch. http://ozonewatch.gsfc.nasa.gov/ monthly/, Mar. 2014.

[2] W. Aigner, S. Miksch, H. Schumann, and C. Tominski. Visualization of time-oriented data. Springer, 2011.

[3] G. Andrienko, N. Andrienko, U. Demsar, D. Dransch, J. Dykes, S. I. Fabrikant, M. Jern, M. J. Kraak, H. Schumann, and C. Tominski. Space, time and visual analytics. International Journal of Geographical Information Science, 24(10):1577-1600, Oct. 2010.

[4] L. Byron and M. Wattenberg. Stacked graphs-geometry \& aesthetics. IEEE Transactions on Visualization and Computer Graphics, 14(6):1245-1252, 2008.

[5] J. Chen, C. Qiao, and R. Zhao. A Voronoi interior adjacency-based approach for generating a contour tree. Computers \& geosciences, 30(4):355-367, 2004

[6] W. Cui, S. Liu, L. Tan, C. Shi, Y. Song, Z. Gao, H. Qu, and X. Tong. TextFlow: Towards better understanding of evolving topics in text. IEEE Transactions on Visualization and Computer Graphics, 17(12):24122421, 2011

[7] Y. Drocourt, R. Borgo, K. Scharrer, T. Murray, S. Bevan, and M. Chen Temporal visualization of boundarybased geoinformation using radial projection. In Computer Graphics Forum, volume 30, pages 981-990. Wiley Online Library, 2011.

[8] G. Ellis and A. Dix. A taxonomy of clutter reduction for information visualisation. IEEE Transactions on Visualization and Computer Graphics, 13(6):1216-1223, 2007.

[9] D. Fisher. Hotmap: Looking at geographic attention. IEEE Transactions on Visualization and Computer Graphics, 13(6):1184-1191, 2007.

[10] M. Frenkel and R. Basri. Curve matching using the fast marching method. In Energy Minimization Methods in Computer Vision and Pattern Recognition, pages 35-51. Springer, 2003.

[11] F. Ge, S. Wang, and T. Liu. Image-segmentation evaluation from the perspective of salient object extraction. In Computer Vision and Pattern Recognition, 2006 IEEE Computer Society Conference on, volume 1, pages 1146-1153. IEEE, 2006.

[12] D. Geiger, A. Gupta, L. A. Costa, and J. Vlontzos. Dynamic programming for detecting, tracking, and matching deformable contours. Pattern Analysis and Machine Intelligence, IEEE Transactions on, 17(3):294 302, 1995.

[13] M. Gleicher, D. Albers, R. Walker, I. Jusufi, C. D. Hansen, and J. C. Roberts. Visual comparison for information visualization. Information Visualization, 10(4):289-309, 2011.

[14] L. Goldmann and T. Adamek, P. Vajda, M. Karaman, R. Mörzinger, E. Galmar, T. Sikora, N. E. O'Connor, T. Ha-Minh, T. Ebrahimi, P. Schallauer and B. Huet. Towards fully automatic image segmentation evaluation. In Advanced Concepts for Intelligent Vision Systems, pages 566577. Springer, 2008.

[15] J. Heer, N. Kong, and M. Agrawala. Sizing the horizon: the effects of chart size and layering on the graphical perception of time series visualizations. In Proceedings of the SIGCHI Conference on Human Factors in Computing Systems (CHI), pages 1303-1312, 2009.

[16] Q. Huang and B. Dom. Quantitative methods of evaluating image segmentation. In Image Processing, 1995. Proceedings, International Conference on, volume 3, pages 53-56. IEEE, 1995.

[17] D. P. Huttenlocher, G. A. Klanderman, and W. J. Rucklidge. Comparing images using the Hausdorff distance. Pattern Analysis and Machine Intelligence, IEEE Transactions on, 15(9):850-863, 1993.

[18] T. Kapler and W. Wright. Geotime information visualization. In Proceedings of the IEEE Symposium on Information Visualization (InfoVis), pages 25-32, 2004.

[19] J. Kleinberg and É. Tardos. Algorithm design. Pearson Education India, 2006.

[20] S. Liu, J. Pu, Q. Luo, H. Qu, L. M. Ni, and R. Krishnan. VAIT: A visual analytics system for metropolitan transportation. IEEE Transactions on Visualization and Computer Graphics, pages 1586-1596, 2013.

[21] M. M. Malik, C. Heinzl, and M. E. Groeller. Comparative visualization for parameter studies of dataset series. IEEE Transactions on Visualization and Computer Graphics, 16(5):829-840, 2010.

[22] D. Martin, C. Fowlkes, D. Tal, and J. Malik. A database of human segmented natural images and its application to evaluating segmentation algorithms and measuring ecological statistics. In Computer Vision, 2001. ICCV 2001. Proceedings. Eighth IEEE International Conference on, vol- ume 2, pages 416-423. IEEE, 2001.

[23] U. Maulik and S. Bandyopadhyay. Performance evaluation of some clustering algorithms and validity indices. Pattern Analysis and Machine Intelligence, IEEE Transactions on, 24(12):1650-1654, 2002.

[24] V. Movahedi and J. H. Elder. Design and perceptual validation of performance measures for salient object segmentation. In Computer Vision and Pattern Recognition Workshops (CVPRW), 2010 IEEE Computer Society Conference on, pages 49-56. IEEE, 2010.

[25] T. Munzner, F. Guimbretire, S. Tasiran, L. Zhang, and Y. Zhou. Treejuxtaposer: scalable tree comparison using focus+context with guaranteed visibility. In ACM Transactions on Graphics (TOG), volume 22, pages 453-462. ACM, 2003.

[26] J. B. Procter, J. Thompson, I. Letunic, C. Creevey, F. Jossinet, and G. J. Barton. Visualization of multiple alignments, phylogenies and gene family evolution. Nature methods, 7:S16-S25, 2010.

[27] G. Robertson, R. Fernandez, D. Fisher, B. Lee, and J. Stasko. Effectiveness of animation in trend visualization. IEEE Transactions on Visualization and Computer Graphics, 14(6):1325-1332, 2008.

[28] J. Schmidt, M. E. Groeller, and S. Bruckner. Vaico: Visual analysis for image comparison. IEEE Transactions on Visualization and Computer Graphics, 19(12):2090-2099, 2013.

[29] C. Scott and R. Nowak. Robust contour matching via the orderpreserving assignment problem. Image Processing, IEEE Transactions on, 15(7):1831-1838, 2006.

[30] P. Shanbhag, P. Rheingans, and M. des Jardins. Temporal visualization of planning polygons for efficient partitioning of geo-spatial data. In Proceedings of the IEEE Symposium on Information Visualization (InfoVis), pages 211-218. IEEE, 2005.

[31] B. Shneiderman. The eyes have it: A task by data type taxonomy for information visualizations. In Visual Languages, 1996. Proceedings., IEEE Symposium on, pages 336-343. IEEE, 1996.

[32] H. D. Tagare, D. O'Shea, and D. Groisser. Non-rigid shape comparison of plane curves in images. Journal of Mathematical Imaging and Vision, 16(1):57-68, 2002.

[33] V. Taimouri and J. Hua. Visualization of shape motions in shape space. IEEE Transactions on Visualization and Computer Graphics, 19(12):2644-2652, 2013

[34] C. Tietjen, B. Meyer, S. Schlechtweg, B. Preim, I. Hertel, and G. Strau. Enhancing slice-based visualizations of medical volume data. In Proceedings of the Eighth Joint Eurographics/IEEE VGTC conference on Vi sualization, pages 123-130. Eurographics Association, 2006.

[35] C. Tominski, H. Schumann, G. Andrienko, and N. Andrienko. Stackingbased visualization of trajectory attribute data. IEEE Transactions on Visualization and Computer Graphics, 18(12):2565-2574, Dec. 2012.

[36] M. Q. Wang Baldonado, A. Woodruff, and A. Kuchinsky. Guidelines for using multiple views in information visualization. In Proceedings of the working conference on Advanced visual interfaces, pages 110-119. ACM, 2000.

[37] C. Ware. Information visualization: perception for design. Elsevier, 2012.

[38] M. Weber, M. Alexa, and W. Müller. Visualizing time-series on spirals. In Proceedings of the IEEE Symposium on Information Visualization (InfoVis), pages 7-7. IEEE Computer Society, 2001.

[39] N. Willems, H. van de Wetering, and J. J. van Wijk. Evaluation of the visibility of vessel movement features in trajectory visualizations. Comput. Graph. Forum, 30(3):801-810, 2011.

[40] J. Zhao, P. Forer, and A. S. Harvey. Activities, ringmaps and geovisualization of large human movement fields. Information Visualization, 7(34):198-209, 2008. 